\title{
Ultrasound-based Sensing Models for Finger Motion Classification
}

\author{
Youjia Huang, Student Member, IEEE, Xingchen Yang, Student Member, IEEE, \\ Yuefeng Li, Student Member, IEEE, Dalin Zhou, Student Member, IEEE, Keshi He, Member, IEEE, and \\ Honghai Liu*, Senior Member, IEEE
}

\begin{abstract}
Motions of the fingers are complex since hand grasping and manipulation are conducted by spatial and temporal coordination of forearm muscles and tendons. The dominant methods based on surface electromyography (sEMG) could not offer satisfactory solutions for finger motion classification due to its inherent nature of measuring the electrical activity of motor units at the skin's surface. In order to recognize morphological changes of forearm muscles for accurate hand motion prediction, ultrasound imaging is employed to investigate the feasibility of detecting mechanical deformation of deep muscle compartments in potential clinical applications. In this study, finger motion classification has been represented as subproblems: recognizing the discrete finger motions and predicting the continuous finger angles. Predefined 14 finger motions are presented in both SEMG signals and ultrasound images and captured simultaneously. Linear discriminant analysis classifier shows the ultrasound has better average accuracy $(95.88 \%)$ than the sEMG $(90.14 \%)$. On the other hand, the study of predicting the metacarpophalangeal (MCP) joint angle of each finger in non-period movements also confirms that classification method based on ultrasound achieves better results (average correlation $0.89 \pm 0.07$ and NRMSE 0.15 $\pm 0.05)$ than sEMG $(0.81 \pm 0.09$ and $0.19 \pm 0.05)$. The research outcomes evidently demonstrate that the ultrasound can be a feasible solution for muscle-driven machine interface, such as accurate finger motion control of prostheses and wearable robotic devices.
\end{abstract}

Index Terms-Ultrasound imaging, surface electromyography, finger motion classification, human-machine interface (HMI), prosthesis control.

\section{INTRODUCTION}

$\mathbf{M}$ ONITORING muscle activities using non-invasive human-machine interface (HMI) is an extremely valuable and challenging task, which can be applied to remote manipulation, muscle rehabilitation and prosthesis control. The core technology is to achieve accurate and stable hand gesture classification using forearm muscle information. With the development of more advanced execution devices such as hand prostheses, more accurate, dexterous and intuitive control is urgently needed [1]-[3]. Many sensor technologies have been applied to this field and are aimed at acting as a

This work is supported by the National Natural Science Foundation of China (Grant No.51575338).

Huang, Yang, Li, He and Liu are with the State Key Laboratory of Mechanical System and Vibration, School of Mechanical Engineering, Shanghai Jiao Tong University, Shanghai, China (e-mail: yjhuang@sjtu.edu.cn, xingchen.yang@sjtu.edu.cn, yflee248@sjtu.edu.cn, lovejessie@sjtu.edu.cn, honghai.liu@sjtu.edu.cn).

Zhou and Liu are with the School of Computing, the University of Portsmouth, PO1 3HE, Portsmouth, UK(e-mail: dalin.zhou@port.ac.uk, honghai.liu@port.ac.uk). Corresponding author: H.Liu. satisfactory HMI, achieving a desirable gesture classification performance [4].

Among all the sensing techniques, sEMG and ultrasound are the most suitable alternatives for hand gesture classification, because of the ability of detecting muscle information of the forearm. sEMG is a kind of predominant technology for prosthesis control, but it has some inherent limitations for instance crosstalk, attenuation, low signal to noise ratio, nonstationarity and lacking specificity for deep muscles [4]. On the contrary, ultrasound can detect morphological changes of both superficial and deep muscles, with a high spatial and temporal resolution [5]. Therefore, ultrasound could be superior for hand gesture classification.

Among all the hand gestures, the finger motion is of vital importance. Because it can provide multi-degree of freedom control which is significant for an intelligent and dexterous execution device. To achieve finger motion control, accurate finger motion classification is a premise. Fig. 1 (a) shows the distribution and structure of the forearm muscles. Among these muscles, flexor pollicis longus, flexor digitorum superficialis, flexor digitorum profundus, extensor digiti minimi, abductor pollicis longus, extensor digitorum and extensor pollicis longus are related to finger movements. Generally speaking, the flexor digitorum superficialis, flexor digitorum profundus and extensor digitorum are the most important muscles for finger-related movements, which control the flexion and the extension of index, middle, ring and little fingers. The movement of the thumb is relatively independent and controlled by the extensor pollicis longus, the flexor pollicis longus and the abductor pollicis longus. Since some finger-related muscles are deep below the skin and sEMG fails to capture deep muscle activity precisely, ultrasound takes some advantages for accurate finger motion classification.

In this paper, the finger motion classification performances for sEMG and ultrasound were compared, including discrete finger motion recognition and continuous finger position estimation. It can be considered as the follow-up to [6], except that more comprehensive situations are considered including a continuous decoding part. It aims at evaluating the ultrasoundbased models for finger motion classification, using the sEMG as the baseline. This article is not aimed at any specific application scenarios, but rather to compare the accuracy and stability of sEMG and ultrasound in the expression of forearm muscle information. To guarantee fairness, sEMG signals and ultrasound images were collected simultaneously during the experiment. 


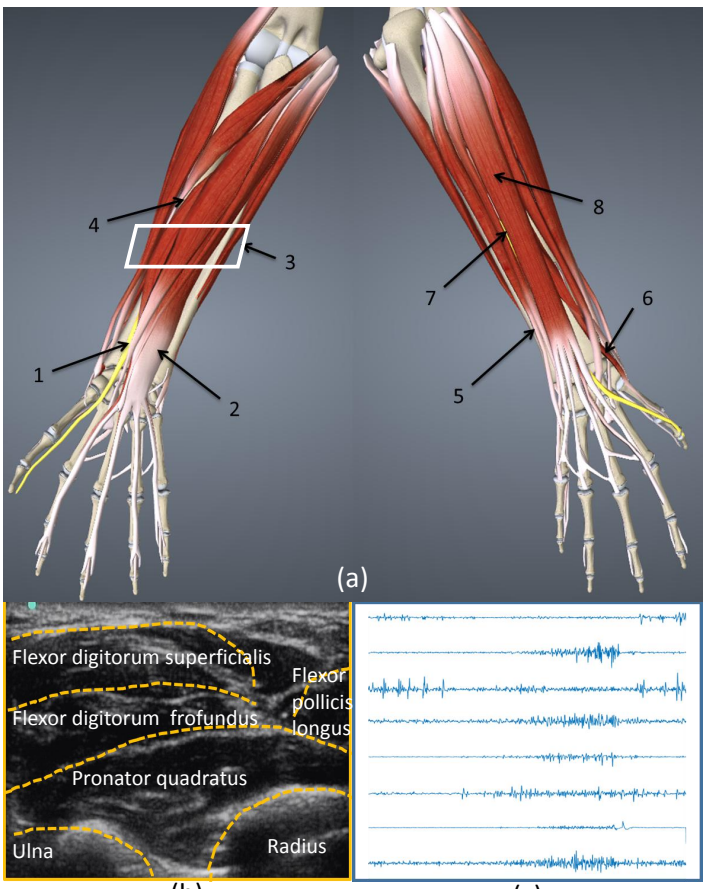

(b)

(c)

Fig. 1. (a) is the distribution and structure of the forearm muscles. (b) is a ultrasound image which demonstrates the cross-section of the forearm muscles in the specific position. (c) is the sEMG signal of 8 channels corresponding to the position labeled in (a)

The study consists of two experiments for the purpose of evaluating the ultrasound/sEMG sensing models for finger motion classification. In Experiment 1, we compared the discrete finger motion recognition accuracies of these two HMIs for 14 different finger motions. In Experiment 2, the continuous decoding property of sEMG and ultrasound for different metacarpophalangeal (MCP) joint angles of each finger were evaluated.

The rest of the paper is organized as follows: first, the related works about sEMG/ultrasound-based gesture classification are introduced in Section II. The details about the experiment setup and the methods used to process the obtained signals are described in Sections III and IV. The experiment results are shown in Section V. Comprehensive discussion is provided for both methodology and future challenges in Section VI. Finally, conclusion is drawn in Section VII.

\section{RELATED WORKS}

\section{A. sEMG-based Gesture Classification}

Since sEMG is closely related to neural signals, it is widely used for hand gesture classification. Many studies about gesture classification based on sEMG has been reported, and various features and classifiers have been compared according to the classification performance [4], [7]-[10].

Duo to the inherent limitations such as lacking sufficient information of deep muscles and crosstalk, it is difficult to recognize deep-muscles-related finger motions for sEMG. However, given the necessity that the finger motions can provide more precise prosthesis control, it still attracts tremendous attentions [7]-[9]. Besides, some practical gestures like hand manipulation, which are highly related to finger motions, have been studied recently [11], [12]. But due to limited information of deep muscles can be captured, the classification performance of finger motion is not desirable enough to be applied in real implement.

A shortcoming of traditional pattern recognition control is that it is unable to provide a continuous and intuitive feeling for the subjects. Jiang et al. achieved a proportional control of wrist movements with multiple degrees of freedom by non-negative matrix factorization [13]. Furthermore, the relationship between the sEMG feature and the finger angle was studied by some researchers [14]-[17]. These studies aim to continuously decode the finger joint angles from sEMG signals. However, as same as discrete finger motion recognition, the performance of sEMG-based continuous decoding cannot meet the realistic requirement.

\section{B. Ultrasound-based Gesture Classification}

In order to overcome the limitations of sEMG and promote the HMI into clinical application, some new HMIs begin to emerge. The ultrasound imaging is regarded as the most promising one because it is non-invasive, safe and has high resolution about the morphological structure of both superficial and deep muscles [5]. These morphological features such as muscle thickness, pennation angle and fascicle length can give a more detailed description of muscle contractions.

Recently, many researchers have started to apply ultrasound to control external devices such as prostheses. Related studies prove that it is feasible for ultrasound to replace sEMG in analyzing muscle activities [18]-[20]. Considering the high resolution of the ultrasound imaging, it is suitable for some dexterous tasks like finger motion classification. Some researchers have studied the finger motion classification using the ultrasound imaging technology and derived promising results [21]-[23].

Besides, Sikdar et al. realized the online classification of hand and finger motions based on ultrasound imaging, which validated the online performance of ultrasound-based HMI. They also investigated the robustness of the ultrasound-based HMI when the arm was in the different positions, proving that it was robust against arm position changing. [24].

Furthermore, Castellini's research focused on predicting the finger position and finger force by ultrasound images and found the linear relationships between a spatial first-order feature, finger position and finger tip force [5], [25].

There are also some studies involving the comparison of the above two HMIs in estimating the wrist angle, the torque of the biceps brachii muscles and finger tip force [26]-[28]. However, so far little is known about their comparative performance for finger motion classification, which is the gap we are addressing in this paper.

\section{DATA ACQUISITION}

\section{A. Experiment Setup}

Eight able-limbed male subjects participated in these experiments (age: 20-24 years old; height: 167-187 cm; weight: $55-75 \mathrm{~kg}$ ). Half of the subjects have the experience on hand 
gesture recognition based on either sEMG or ultrasound, while the other half do not. The experiment setup is shown in Fig. 2. The data collection during the experiments included ultrasound images, sEMG signals and MCP joint angles, the latter was for Experiment 2 only. For ultrasound, a clinical portable ultrasound system Mindray DP-50 with a $38 \mathrm{~mm}$ 7.5 MHZ central frequency linear transducer 75L38EA was employed. The ultrasound images produced by the system were captured through a commercial video capture card (TC$540 \mathrm{~N} 1)$. The final video with a $30 \mathrm{~Hz}$ frame rate was saved in the computer for a further analysis. Trigno Wireless System (Delsys Inc., USA) was used to collect sEMG signals. The sampling frequency was set to $2000 \mathrm{~Hz}$. Furthermore, in Experiment 2, a 5DT Data Glove (5DT Inc., USA) with 14 strain gauges was employed, and 5 corresponding strain gauges were used to record the MCP joint angles of each finger.

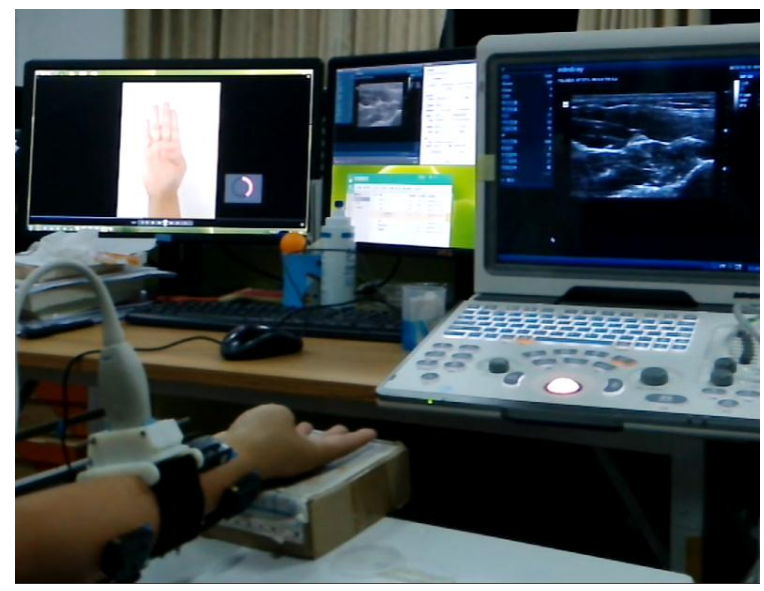

Fig. 2. Experiment setup. The subjects would perform different finger motions following a prompt video in Experiment 1. The difference in Experiment 2 is that the subjects were asked to wear a data glove to collect the MCP joint angles.

Considering the fairness of the comparison, both the sEMG sensors and the ultrasound probe were placed on the forearm to ensure simultaneous data acquisition. All the superficial finger-related muscles were selected at first, then eight sEMG sensors were used and each SEMG sensor was attached on the skin above the corresponding muscle. As each EMG sensor was placed, its position was adjusted to make sure the sEMG signals from corresponding muscle could be properly detected. The eight sEMG sensors were attached on the skin above the following muscles: flexor pollicis longus, flexor digitorum superficialis, flexor digitorum superficialis, flexor digitorum profundusa, extensor digiti minimi, extensor digitorum, abductor pollicis longus and extensor pollicis longus [14], [15]. When attaching the sEMG sensors, the position of ultrasound probe was taken into consideration. The probe was fixed on the subjects' forearm which was approximately $6 \mathrm{~cm}$ away from the wrist and perpendicular to the ulna using a customized cuff. The details about the sensor placement are shown in Fig. 3.

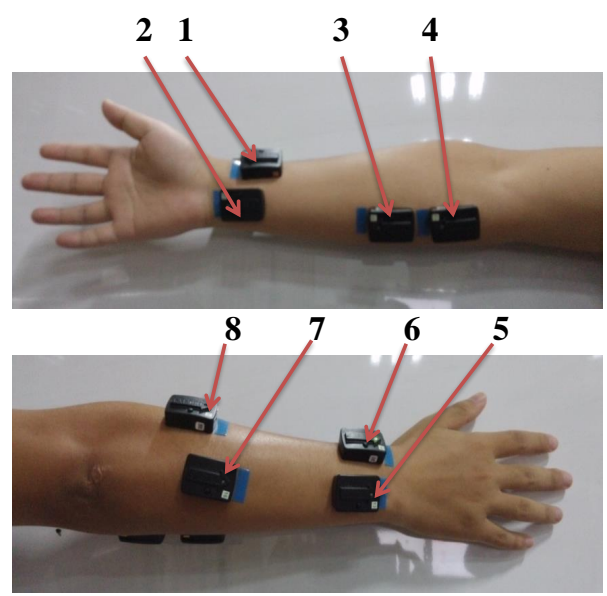

Ultrasound probe

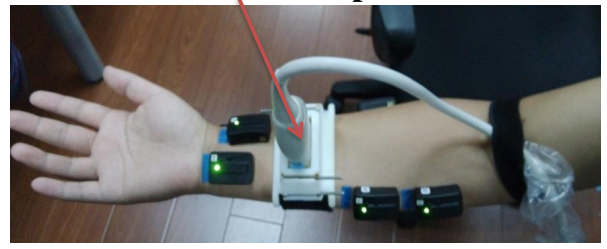

Fig. 3. The placement of the sEMG sensors and ultrasound probe.

\section{B. Experiment Scenarios}

1) Experiment 1: Discrete Finger Motion Recognition: In this experiment, the subjects were asked to sit in front of two computer screens. One was used to instruct the subjects to complete corresponding finger motions while the other was used to show the acquired ultrasound images and sEMG signals. Initially, the forearm of each subject was cleaned with medical alcohol. Then eight sEMG sensors were attached to the predefined positions. Before fixing the ultrasound probe, ultrasound gel was applied between the probe and the skin to reduce acoustic impendence between them. The subjects were instructed to hold their forearms supine on the table in order to avoid probe shifting with the influence of gravity. Finally, the predefined 14 finger motions including rest state in Fig. 4 were performed. There were 10 trials' experiments in total. In each trial, the subjects were required to perform above 14 different motions once. Each motion was held for 5 seconds followed by a 5 -second rest. After completing the first 5 trials, the subjects took a 5 minutes' rest to avoid muscle fatigue.

2) Experiment 2: Continuous Decoding of MCP Joint Angles: The preparation process of the Experiment 2 was nearly as same as Experiment 1. The difference was that the subjects were required to wear a data glove. During this experiment, the subjects were asked to move their five fingers with instruction. Each finger's movement was non-periodic and lasted for 60 seconds, between various finger movements, there were $2 \mathrm{~min}$ utes' rest time to avoid muscle fatigue. The ultrasound images, sEMG signals and the MCP joint angles were collected for further analysis simultaneously. 


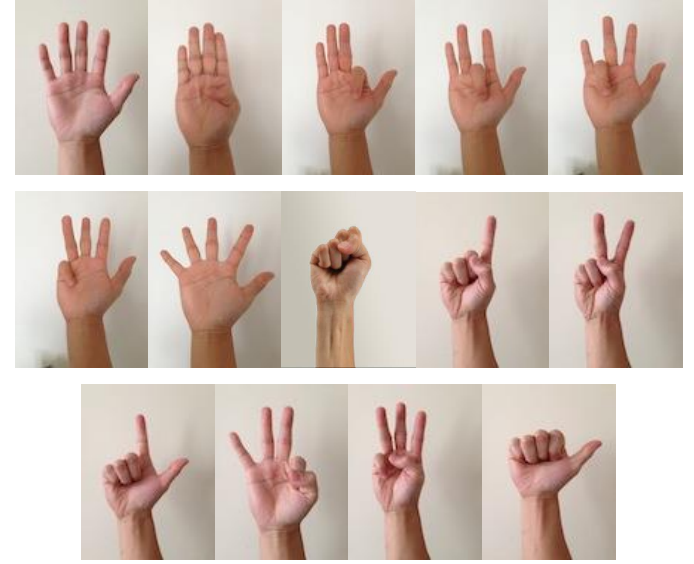

Fig. 4. The experimental paradigm, including 5 single finger motions, 2 double finger motions, 2 triple finger motions, 2 four finger motions, hand open, fist and rest.

\section{Methodology}

\section{A. Synchronization between Different Signals}

At the stage of data acquisition, it was hard to ensure that all the equipment started to gather data simultaneously, which would have significant effect on predicting the MCP joint angles of each finger. To solve this problem, the subjects were asked to fist five times quickly at the beginning of Experiment 2, when the ultrasound images, sEMG signals and glove data were collected. Preprocessing was applied to extract the features of ultrasound images and SEMG signals as introduced in section IV-B. After normalization and resampling, the cross-correlations between MCP angles of middle finger and the principal components of the sEMG features, ultrasound features were estimated respectively based on Equation 1 [29].

$$
\hat{R}_{x y}(m)= \begin{cases}\sum_{n=0}^{N-m-1} x_{n+m} y_{n}^{*}, & m \geq 0 \\ R_{y x}(-m), & m<0\end{cases}
$$

Where $x$ and $y$ are two different signals, $N$ is the length of the signal and the asterisk denotes complex conjugation. Cross-correlation is a widely used method in signal processing to determine the delay time of two signals. The coordinate of the maximum value in cross-correlation $\hat{R}_{x y}$ is related to delay time between the signals as shown in Equation 2 . The frequency represents the frequency of the signal after resampling.

$$
\text { delay }=\{\underset{m}{\arg \max } \hat{R}(m)\} / \text { frequency }
$$

A specific subject's example is shown in Fig. 5. Initially, the cross-correlation between sEMG features' principal component and the glove data, the ultrasound image features' principal component and the glove data were calculated respectively. Latter, Equation 2 was used to determine the delay. For this subject, the EMG signals were 1.24 seconds behind the glove data while the glove data was 0.29 seconds behind the ultrasound images. This method was acceptable for eliminating the primary time-delay between signals in the Experiment 2. It is noteworthy that time-calibration is needed only once for each subject, because the data of three sensors is continuously collected without a break no matter during finger movements or rest.
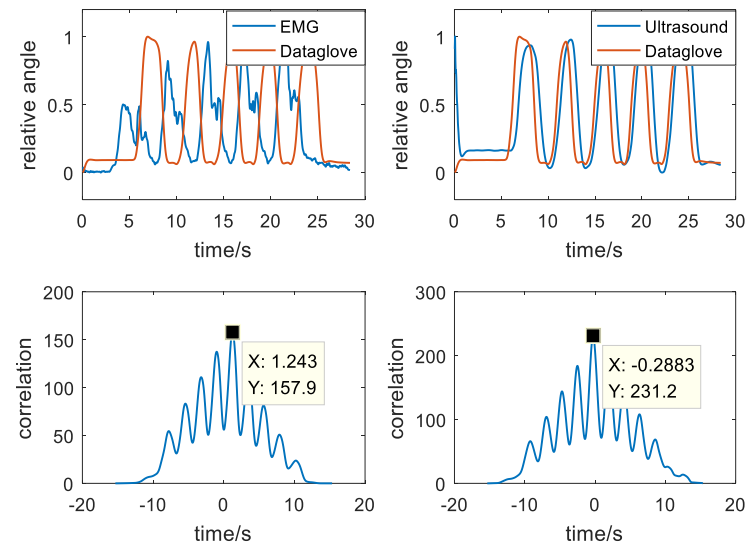

Fig. 5. Time delay between sEMG signals and glove data, ultrasound images and glove data. The upper two figures show the glove data and the signals' principal component after resampling, and the lower two figures show the cross-correlation results between them.

\section{B. Feature Extraction}

1) sEMG Feature Extraction: Feature extraction is a crucial step for pattern recognition. For sEMG based hand gesture recognition, there are many kinds of feature extraction methods [4]. TD features applied in this study were first proposed by Hudgins $e t$ al. and have been proved to be features with superior classification performance [30]-[32]. Initially, the raw sEMG signals were segmented into a series of moving windows with 200 milliseconds' length and 50 milliseconds' step. Latter, the TD features, namely, mean absolute value (MAV), number of zero crossings (ZC), waveform length (WL) and number of slope changes (SSC) were extracted from each window. Since eight sEMG sensors were used and the features were concatenated together, the feature vector of sEMG signals was 32 dimensions. Each motion lasted for 5 seconds and 2 seconds' signals were selected as stable signals for each finger motion. Eight subjects participated in the experiment. There were 10 trials for each subject and 14 finger motions in each trial. Considering the rest state, which was regard as one kind of motion, accounted for half of the experimental time, the whole number of samples were $26 \times 10 \times 36$.

As for Experiment 2, the MAV feature was extracted from the sEMG signals. The window length and the overlap length were the same as Experiment 1. Both the features and the angle data were filtered by applying a 2nd order Butter-worth filter with a $10 \mathrm{~Hz}$ cut-off frequency [14]. Then, the sEMG features and the MCP joint angles of each finger were resampled to the same frequency. The time delay between features and MCP joint angles can be eliminated by the method mentioned in section IV-A. 
2) Feature Extraction of Ultrasound Images: In the field of ultrasound-based HMI, there are mainly two feature extraction methods. The first method is to extract features from the image sequences, for instance optical flow and correlation methods [21] [22]. The second approach is to extract features from the static ultrasound images such as a spatial first-order feature [5]. The B-mode ultrasound probe consisted of multiple transducer elements, each element in the linear probe of the B-mode equipment can be regarded as a single-element transducer used in A-mode ultrasound. In addition, using the whole ultrasound images to classify finger motions seemed redundant, because the different parts of the image were highly related because of synergic movement of muscles. Hence, the feature used in this study did not focus on the whole image, but only 5 columns of the image were chosen, which were uniformly selected from the original ultrasound image. The data of each column of the B-mode ultrasound image can be considered as the data derived from A-mode ultrasound. By this means, the data used in this paper can be considered as A-mode ultrasound signal.

An external video graphics array (VGA) connector was applied to grab the ultrasound video from adopted B-mode ultrasound system. Initially, the unrelated area in the video was eliminated in order to obtain the image sequences which only contained the ultrasound images with respect to the forearm muscles. The color images were then transformed to gray images and the gray values were scaled to the range of 0 1 for further processing.

As mentioned in sEMG signals preprocessing, 36 images were uniformly selected from the 2 nd second to the 4th second in a 5-second motion period. Latter, five columns' data from each selected ultrasound image was chosen evenly. Each column's data was segmented into several windows with a segmentation-length of 20 pixels. In each window, the linear fitting was used for fitting the data. The fitting coefficients were noted as the features. Hence the whole sample number of ultrasound images was as same as sEMG signals. The whole feature extraction flow chart is shown in Fig. 6.

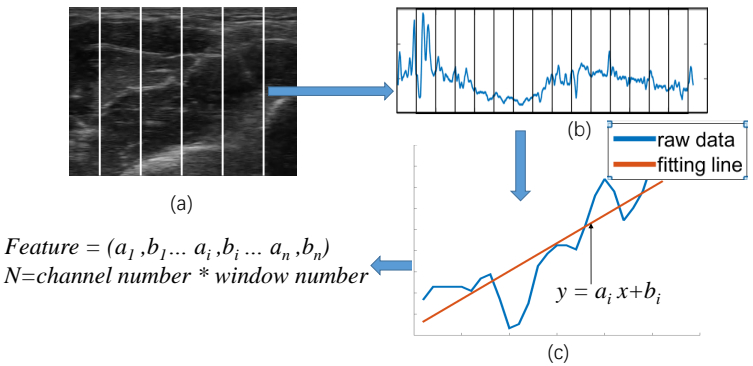

Fig. 6. The overall schematic of ultrasound image feature extraction.

In Experiment 2, the feature extraction process of ultrasound images was almost the same as that in Experiment 1. The feature vectors of ultrasound images and the finger angles from data glove were both filtered by a 2nd order butterworth filter with a cut-off frequency of $10 \mathrm{~Hz}$. The feature vectors were resampled to the same frequency and the time delay was removed. Finally, principal component analysis (PCA) was applied to reduce the dimension of original feature space to 12 dimensions. All the procedures of data processing in this experiment were performed off-line. The raw data was separated into multiple windows and the features were extracted from segmented windows. The data were divided into two sections, one was for training the classifier model and the other was for testing. For Experiment 2, before the feature vectors were transferred to the classifier model, filtering, resampling and synchronization process were conducted.

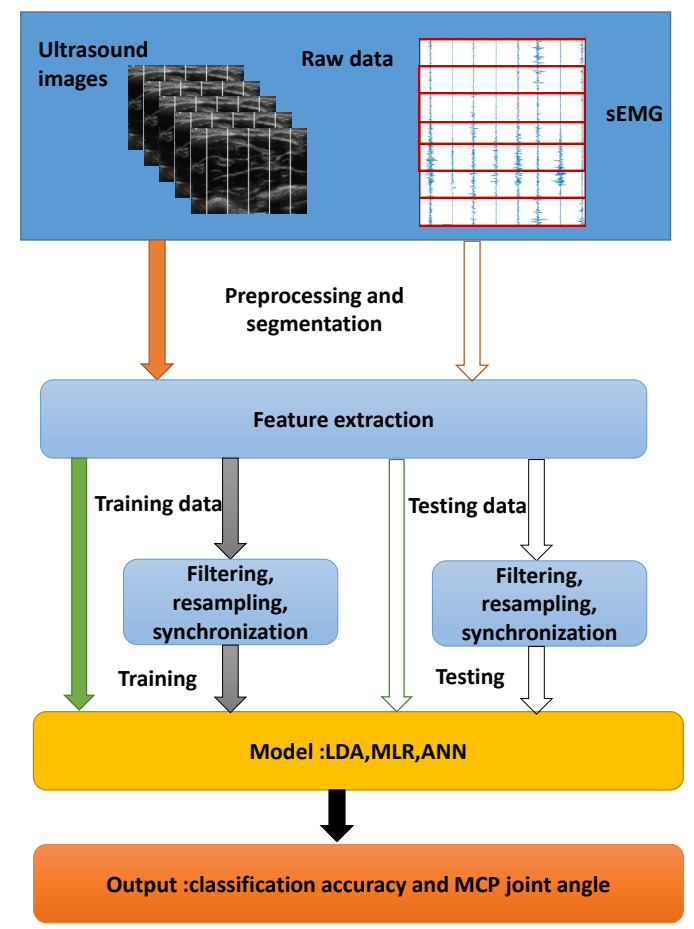

Fig. 7. Overall algorithm of sEMG and ultrasound signal processing.

\section{Classification and Regression}

1) Classification of Discrete Finger Motions: LDA is one of the most commonly used classifiers in myoelectric pattern recognition. It has been proved to be one of the most effective classifiers in this area [31], [32]. Considering the relative fairness of evaluation on ultrasound and sEMG, the LDA classifier was used in pattern recognition both for ultrasound images and sEMG signals. In this part, 2-fold cross-validation was used for the data being used in this research. The crossvalidation was repeated 50 times. For each time, 5 trials data was randomly chosen from the whole data set (both for ultrasound data set and sEMG data set) to train the classifier, and the remaining data was used for testing. The overall average classification accuracy (CA) of the 50 times was then used to evaluate the performances of ultrasound and sEMG. The CA used in this study was the ratio of the number of correctly classified samples to the number of the whole testing samples, defined as follows:

$$
C A=\frac{\text { number of correctly classified samples }}{\text { number of the whole test samples }} \times 100 \%
$$


2) Regression of Continuous MCP Joint Angles: Artificial neural network (ANN) is the most commonly used method for continuous finger movement decoding in sEMG [15]-[17]. Castellinis et al. proved that there was a significant linear relationship between the spatial first-order feature of B-mode ultrasound images and the finger angles according to a multiple linear regression (MLR) [5]. Both the two regression methods were used in this study.

The entire 60-second data for each finger was separated into two parts. The first 30 -second data was used to train the model and the remaining 30-second data was used to evaluate the model. The evaluation criteria of the regression were root mean square deviation (NRMSE) and the correlation coefficient between predicted finger MCP joint angles $\hat{y}$ and the angles $y$ measured by the data glove. The NRMSE and the correlation coefficient are defined in Equations 4 and 5.

$$
\begin{gathered}
\text { NRMSE }=\frac{\sqrt{(\hat{y}-y)^{T} \times(\hat{y}-y) / n}}{y_{\max }-y_{\min }} \\
\text { correlation }_{y \hat{y}}=\frac{\sum_{i=1}^{n}\left(y_{i}-\bar{y}\right)\left(\hat{y}_{i}-\overline{\hat{y}}\right)}{\sqrt{\sum_{i=1}^{n}\left(y_{i}-\bar{y}\right)^{2} \sum_{i=1}^{n}\left(\hat{y}_{i}-\bar{y}\right)^{2}}}
\end{gathered}
$$

\section{Statistical Analysis}

In Experiment 1, one-way ANOVA was used to analyze the performance of various signals in terms of the CA of the 14 discrete finger motions. In Experiment 2, a two-way ANOVA was used to evaluate the regression accuracy, the factors were regression methods, linear regression and neural network regression, and the signal types (sEMG and ultrasound). The significance level of all tests was set as $\mathrm{p}<0.05$.

\section{EXPERIMENT RESULTS}

\section{A. Experiment 1: Discrete Finger Motion Recognition}

The CA for the eight subjects over 14 different finger motions is shown in Fig. 8. The average CA for all the subjects is $95.88 \%$ for ultrasound and $90.14 \%$ for sEMG. One-way ANOVA analysis demonstrates that the result of the ultrasound based method significantly outperforms sEMG base method $(\mathrm{p}=0.009)$. The standard deviation (SD) of the ultrasound for each subject is clearly lower $(p=0.005)$ than that of the sEMG, which indicates that the ultrasound approach performs more stable for the recognition of different finger motions. Furthermore, the result also shows that there is a significant difference between four skilled subjects (subject 1, 3, 6 and 8) and four inexperienced subjects (subject 2,4,5 and 7) for both the sEMG and ultrasound based pattern recognition.

The CA confusion matrixs of the sEMG and ultrasound are given in Fig. 9 and Fig. 10. One-way ANOVA analysis demonstrates that for each finger motion, the $\mathrm{CA}$ of the ultrasound is obviously higher than that of the sEMG. The motion class from 1 to 14 is corresponding to the experimental paradigm in Fig. 4. Experimental result represents that the accuracies of thumb flexion and the little finger flexion are relatively lower for both ultrasound and sEMG. The motion

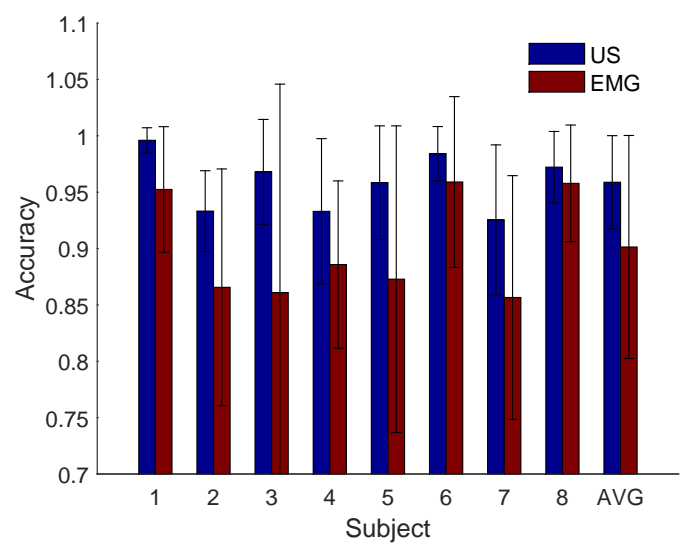

Fig. 8. Classification accuracy of the ultrasound (US)-based and sEMG-based HMI for each subject. The height of the bars denotes the average classification accuracy of 14 different finger motions for each subject while error bars represent the standard deviation.

pairs whose difference is related to the little finger or thumb have a higher confusion error rate, such as the pair consisting of class 2 and class 13 .

As for ultrasound, the probe width of the ultrasound system is $38 \mathrm{~mm}$. The muscles, corresponding to thumb and little finger movements, are towards the periphery of the visual field of the imaging transducer. What's more, the morphological changes of the muscles related to thumb and little finger is relatively small. On the other hand, it is hard to detect the sEMG signals of the deep muscles, as there is a crosstalk between different muscles. The related sEMG signals of little finger or thumb are relatively weaker. However, there might exist some relationship between the muscle morphological changes and SEMG signals since the distribution of confusion error rates of different motions is a little similar for ultrasound and sEMG.

\section{B. Experiment 2: Continuous Decoding of MCP Joint Angles}

1) Signal Correlation: Fig. 11 shows that the correlation coefficients of the predicted and actual MCP joint angles. When using sEMG and MLR, sEMG and ANN, ultrasound and MLR, and ultrasound and ANN, the correlation coefficients are $0.75 \pm 0.11,0.81 \pm 0.09,0.86 \pm 0.08$ and $0.89 \pm$ 0.07 respectively. A two-way ANOVA analysis demonstrates that both the regression methods, MLR or ANN, and the signal types, ultrasound or sEMG, have a significant effect on the prediction accuracy. The $p$ value for regression methods is 0.016 which implies that the ANN performs significantly better than the MLR. The ultrasound makes a more precise prediction than its counterpart $(\mathrm{p}=0.007)$. The $p$ value of interaction is 0.0001 which indicates that the combination of ANN and ultrasound can obtain the best regression performance in this situation. Compared with the MLR, the ANN can deal with some nonlinear situations and it is more robust since the regularization is considered in the model.

2) NRMSE: Fig. 12 shows the average normalized root mean square error (NRMSE) of the predicted and actual MCP joint angles under four different situations: sEMG and MLR, 


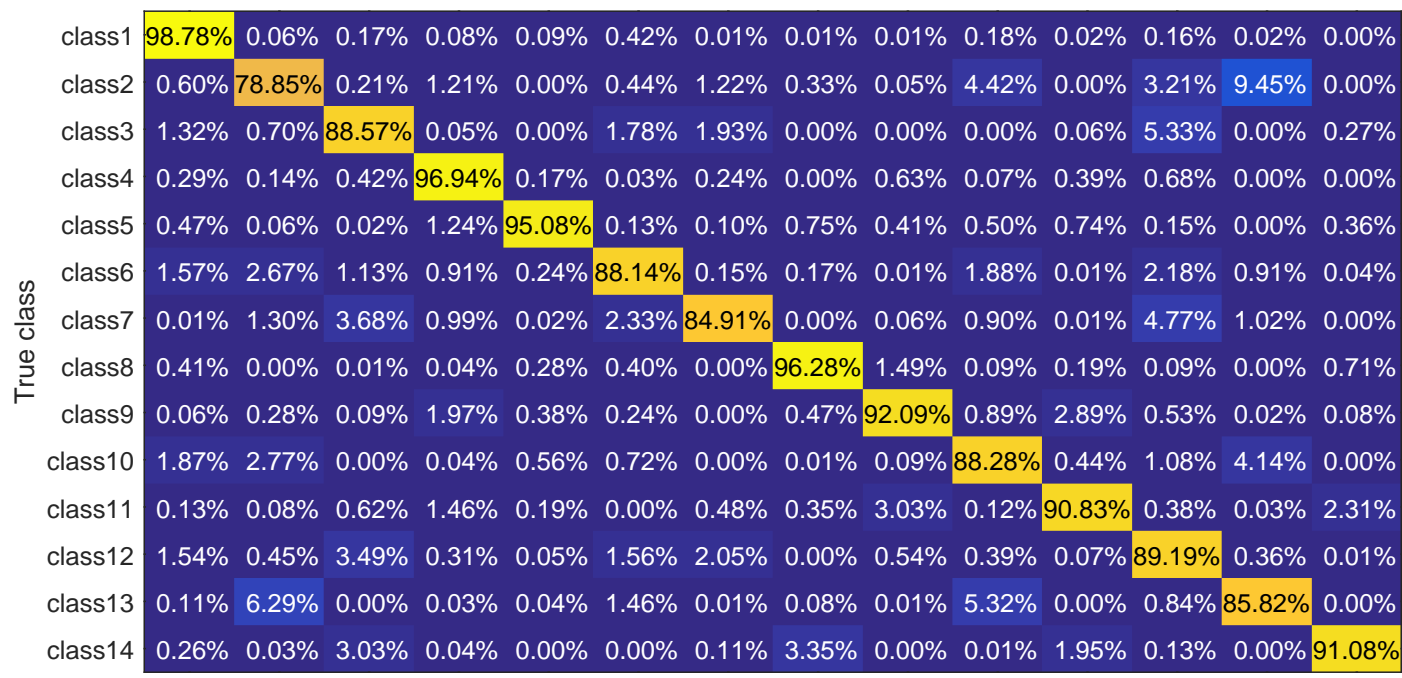

class 1 class 2 class 3 class 4 class 5 class 6 class 7 class 8 class 9 class 10 class 11 class 12 class 13 class 14 Predicted class

Fig. 9. Classification accuracy confusion matrix of 14 different finger motions for sEMG, using LDA classifier and 2-fold cross-validation. The motion class 1 to 14 are corresponding to the experimental paradigm in Fig. 4. The row of each element in the confusion matrix denotes the actual class while the column indicates the predicted class.

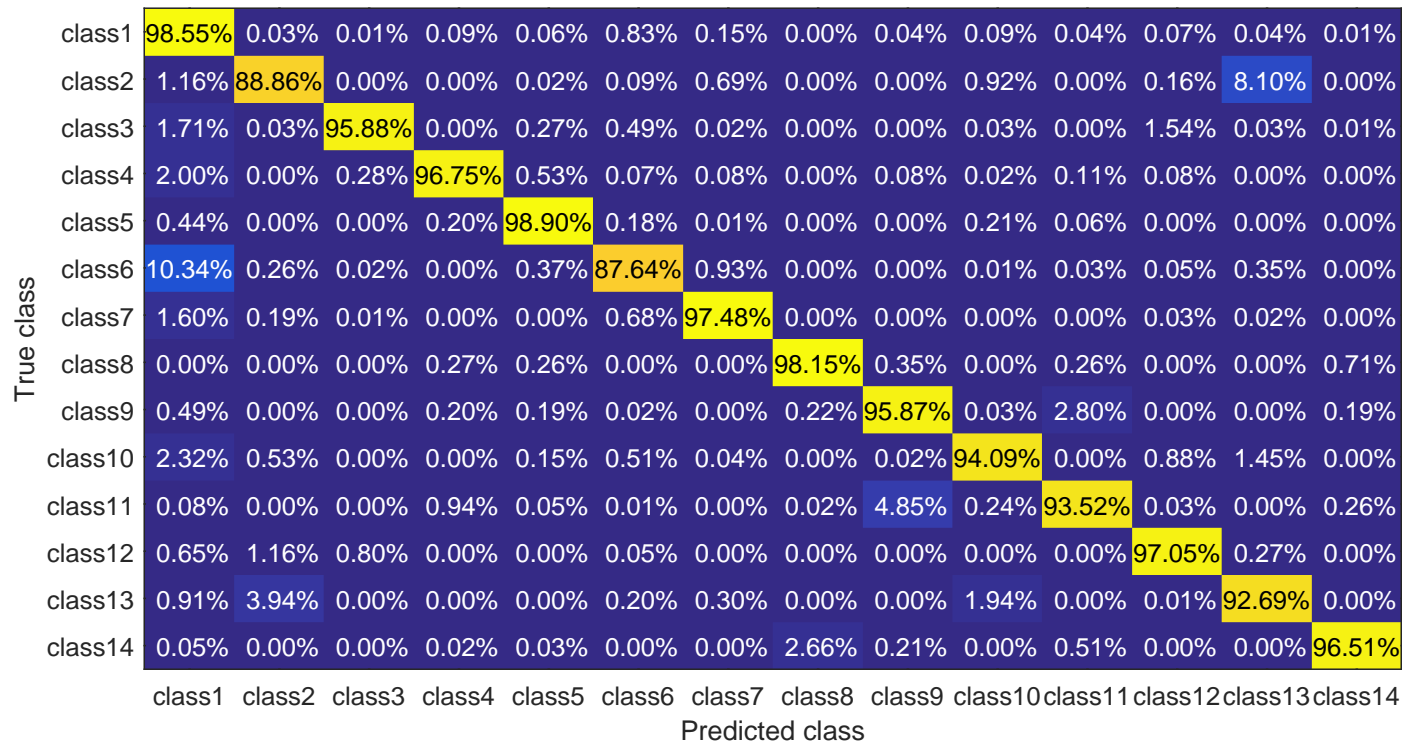

Fig. 10. Confusion matrix of classification accuracy for ultrasound-based HMI. The motion from 1 to 14 are corresponding to the experimental paradigm in Fig. 4.

sEMG and ANN, ultrasound and MLR, and ultrasound and ANN. The NRMSE under these four conditions are $0.22 \pm$ $0.05,0.19 \pm 0.05,0.19 \pm 0.08$ and $0.15 \pm 0.05$, respectively. Two-way ANOVA analysis demonstrates that both the regression methods and the signal types significantly affect the value of the NRMSE. The $p$ values for regression methods, types of signals and the interaction are 0.011, 0.002 and 0.0002, respectively. This indicates that the ANN performs better than the MLR and ultrasound outperforms sEMG. When using ultrasound together with ANN, the NRMSE can get the mini- mum value. Fig. 13 shows the predicted and actual MCP joint angles of the index and little fingers for subject 8 with ANN. The correlation coefficients and NRMSE values of the four sub-figures are 0.93 and 0.10 for Ultrasound_index, 0.86 and 0.13 for $s E M G \_$index, 0.89 and 0.15 for Ultrasound_little, and 0.67 and 0.27 for Ultrasound_little, respectively. 


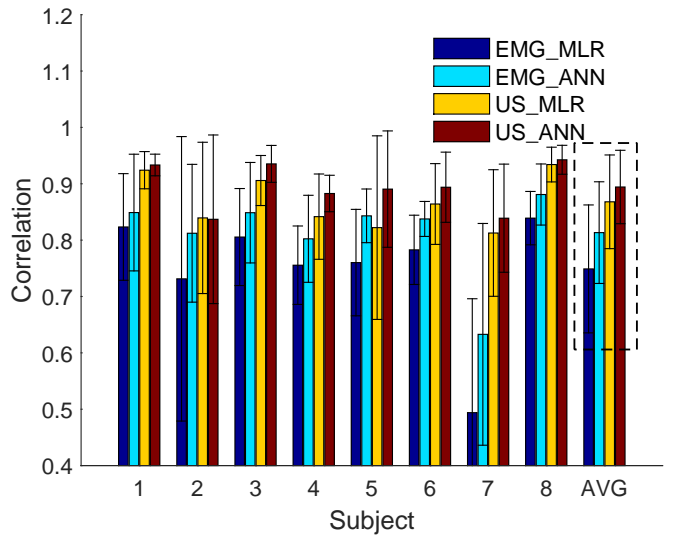

(a)

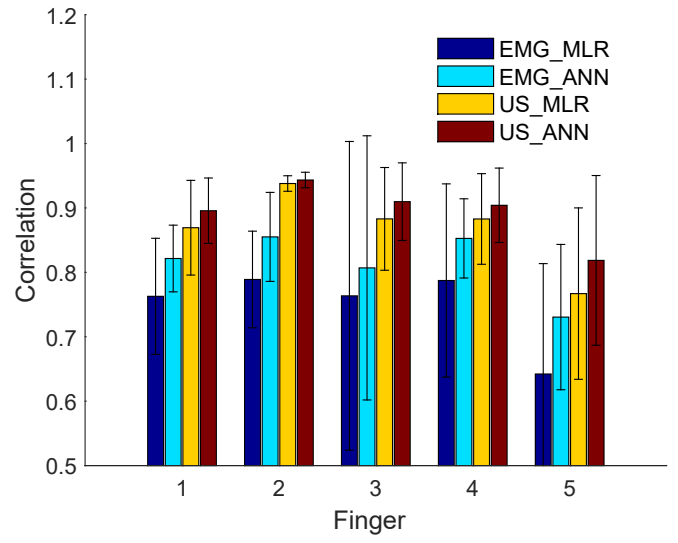

(b)

Fig. 11. The correlation coefficient of the predicted and actual joint angles. The different color bars denote the correlation and its standard deviation under four different situations: sEMG and MLR, sEMG and ANN, ultrasound and MLR, and sEMG and ANN. The X-axis label represents the eight different subjects (a) and five different MCP joint angle of the fingers (b) respectively.

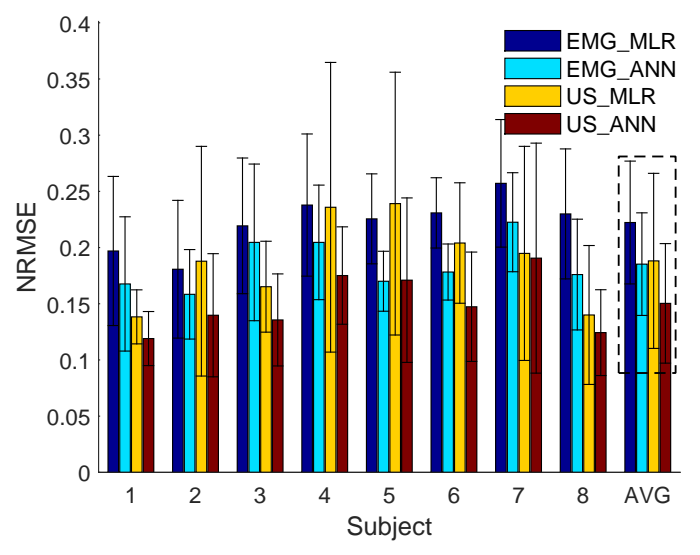

(a)

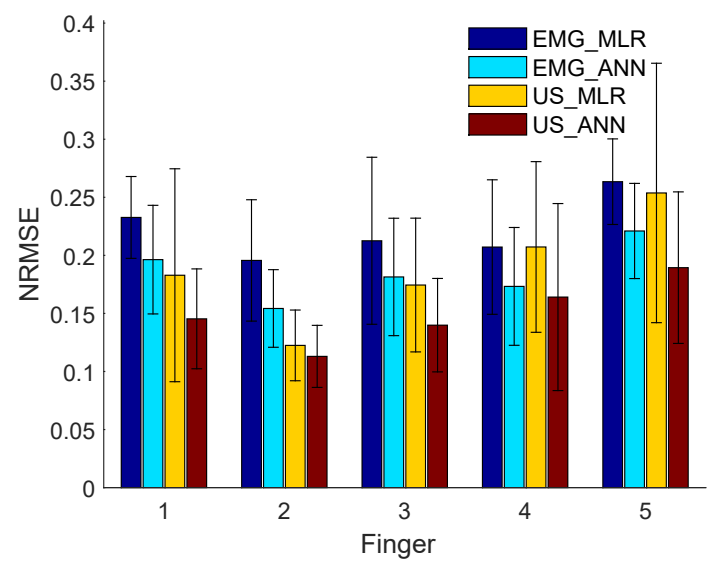

(b)

Fig. 12. The NRMSE of the predicted and actual joint angles for sEMG and ultrasound. The different color bars denote the NRMSE and its standard deviation under four different situations described above. The X-axis label represents the eight different subjects (a) and five different MCP joint angles of the fingers (b) respectively.

\section{DISCUSSIONS}

\section{A. Experiment Design}

The aim of this study is to evaluate the performance of ultrasound-based HMI in finger motion recognition in comparison with the dominant sEMG-based methods. The study is given priority to the classification of the more dexterous hand gestures. The design of this experiment ensures the fairness of the evaluation by collecting both the sEMG signals and the ultrasound images simultaneously in the same situation, avoiding the influence of some irrelevant factors. The interference between these two kinds of sensors is also proved to be non-existent. When attaching the sEMG sensors at first, we found there was no obvious changes for sEMG signals whether the ultrasound probe was used or not, and vice versa. This phenomenon may imply that these two kinds signals can be used in some special fields such as sensor fusion or feedback of the actual control. For example, ultrasound imaging can be used to classify hand gestures while sEMG can be used for force estimation. The placement of sEMG electrodes and ultrasound probe may be impractical in some situations, while this study focuses on the comparison of monitoring the muscle activities of these two methods, the placement of the sensors give the priority to sEMG and it's completely fair for sEMG. On the other hand, considering the completeness of the evaluation, the whole experiment in this study is decomposed into two part: recognizing the discrete finger motions and predicting the continuous finger angles.

Recently, Zheng et al. and Sikdar et al. have studied the discrete finger motion recognition based on the ultrasound images [21], [22], and Castellini et al. have done some researches on continuous finger angle prediction using ultrasound [5]. However, these researches didn't give a comprehensive comparison of ultrasound and sEMG based HMIs, to strength the superiority of the ultrasound-based HMI in prosthesis control. Moreover, the hand gestures selected in the past researches 




Fig. 13. The predicted and actual MCP joint angles of the index and little fingers for subject 8 . The correlation coefficients and NRMSE values of the four sub-figures are 0.93 and 0.10 for Ultrasound_index, 0.86 and 0.13 for sEMG_index, 0.89 and 0.15 for Ultrasound_little, 0.67 and 0.27 for Ultrasound_little, respectively.

were always related to single finger motion. Finger motions such as hand grasping and manipulation, which were always conducted by spatial and temporal coordination of forearm muscles, were complex. The finger motions used in this study contained not only single finger flexion but also combined finger motions. In Sikdar's recent research, more hand gestures have been taken into consideration, but his study focused on the situation that the elbow was in different positions [24]. All the finger-related muscles in the forearm were involved in the experiment paradigm designed in this study. Therefore, this experiment provides a fair and comprehensive comparison of ultrasound and sEMG based HMIs for not only single finger flexion but also combined finger motions recognition.

\section{B. Methods}

All the data processing procedures were designed as similar as possible for both the sEMG and ultrasound in order to ensure the experiment results to be comparative, although there may be some unavoidable alterations when some motion changes happened in the feature extraction stage.

Compared with recent researches, the feature extraction method mentioned in this paper is more straightforward, some columns are chosen from the original images as the input data. A selection is carried out for optimal the channel number and the window length in the feature extraction stage for ultrasound images as showed in Fig. 14. This indicates that the more the channels are, the shorter the window length is, the higher the CA is. This may indicate that the different parts of the ultrasound image are relevant to certain extent and using the whole image for the hand gesture classification seems a little redundant. Using part of the original image can help reduce the computation time, which is particularly important for the online classification. Although Sikdar et al. has realized the real-time hand motion classification, the 200 milliseconds' image collection time and 79 milliseconds' feature extraction time cannot reach a desirable online performance as sEMG

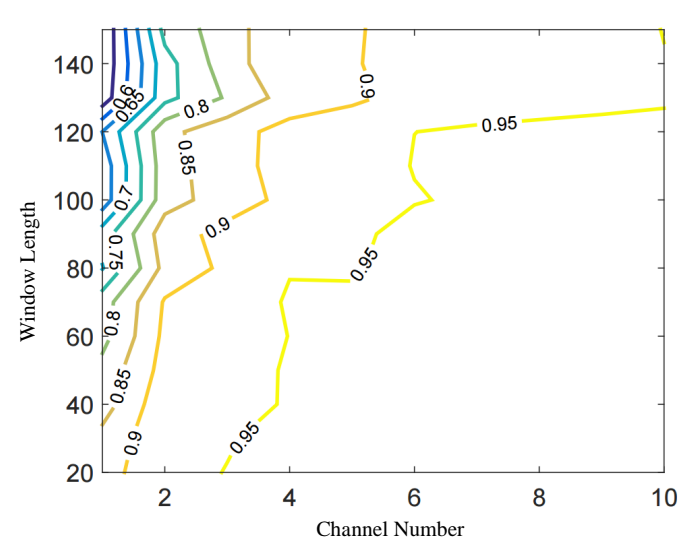

Fig. 14. The classification accuracy changes according to different channels and window lengths.

[24]. With $\mathrm{C}++$, the feature extraction time of each ultrasound image was only $2 \mathrm{~mm}$ in this study, which was faster than the method mentioned in [24]. Although in real-time experiment, more channels are needed for ultrasound-based HMI in consideration of the robustness, the computation time is still not an obstacle for the contemporary computers and it is possible to achieve the same property as the sEMG [33]. More importantly, all the methods both for discrete finger motion recognition and finger angle prediction used in this study are completely able to be applied in online situation. Further work has been targeted in our current research plan.

\section{Future Challenges}

The ultrasound-based HMI has some limitations such as probe shifting before applying to practical scenarios or clinical applications.

1) Probe shifting: During the experiment, it was found that the shift between the ultrasound probe and the skin significantly influenced the accuracy of the classification results. Such a phenomenon was also observed by some other researchers [5], [24]. The shift of the sensors was also a big obstacle for the sEMG. Some algorithms, used to eliminate the effects of electrodes shift in high density sEMG signals, may be applied to ultrasound-based HMI. Spatial features of the high density sEMG signal were employed to solve the problems such as the shift of the electrode, noise and fault of channels [34], [35]. These spatial features could be applied in ultrasound image processing since the linear probe (B-mode device) made up of many single-element transducers can be regard as high density sensors. What's more, if a suitable feature robust against the shift, rotation and scaling of the image could be found, a robust ultrasound-based HMI could be achievable.

2) Wearable ultrasound devices: Although there are already some available commercial wearable medical ultrasound devices, high quality wearable ultrasound devices are required for more dexterous finger motions. In Sikdar's paper, a singleelement mechanical sector probe was introduced in the size of a pen [22]. It could be connected to PC via USB. When it comes to array probes, GE has a handheld ultrasound device 
VSCAN, which integrates the probe and its backend in the size of a smartphone. Philips Lumify is a new comer in this field, which provides a linear array probe subscription service. The probe can be connected to any Android phones and ultrasound images are displayed directly on the phone. Konica Minolta Sonimage P3 is another handhold ultrasound machine in a similar size, with limited choices of probes. All of the devices mentioned above are able to provide high-quality B-mode ultrasound images and can be wearable. In our research group, a wearable ultrasound device is under development, which can use multiple large-scale single element transducers (A-mode ultrasound transducer) to inspect muscle information. By this means, the data processing can be simplified and the system can be wearable and cost-effective [23].

3) Classification robustness: The problem of gesture classification robustness will be focused on in future as well, which is crucial in clinical application. To achieve robust finger motion recognition, various features and classifiers will be tried, and deep learning method will be taken into consideration, which has been applied in tumor classification with a small ultrasound dataset [36].

\section{CONCLUSION}

In this study, we evaluated the performance of the sEMGbased HMI and the ultrasound-based HMI in terms of finger motion classification. The results have demonstrated that the ultrasound performed significantly better than the sEMG in such a more dexterous and accurate control. Further, we have discussed the problems of the ultrasound-based HMI in detail and provided some feasible suggestions on its further exploration. This study confirms that the ultrasound-based HMI has the potential to replace the sEMG-based HMI, especially for more accurate control and dexterous manipulation.

\section{REFERENCES}

[1] M. S. Johannes, J. D. Bigelow, J. M. Burck, S. D. Harshbarger, M. V. Kozlowski, and T. Van Doren, "An overview of the developmental process for the modular prosthetic limb," Johns Hopkins APL Technical Digest, vol. 30, no. 3, pp. 207-216, 2011.

[2] Pan-American Health Organization, International Federation for Medical and Biological Engineering, and Institute of Electrical and Electronics Engineers, Eds., 2009 Pan American health care exchanges (PAHCE 2009): Mexico City, Mexico, 16 - 20 March 2009 ; [conference, workshops, and exhibits, cooperation/linkages]. Piscataway, NJ: IEEE, 2009.

[3] C. Lake and R. Dodson, "Progressive upper limb prosthetics," Phys. Med. Rehabil. Clin. N. Am., vol. 17, no. 1, pp. 49-72, 2006.

[4] Y. Fang, N. Hettiarachchi, D. Zhou, and H. Liu, "Multi-Modal Sensing Techniques for Interfacing Hand Prostheses: A Review," IEEE Sensors Journal, vol. 15, no. 11, pp. 6065-6076, Nov. 2015.

[5] C. Castellini, G. Passig, and E. Zarka, "Using Ultrasound Images of the Forearm to Predict Finger Positions," IEEE Transactions on Neural Systems and Rehabilitation Engineering, vol. 20, no. 6, pp. 788-797, Nov. 2012.

[6] Y. Huang and H. Liu, "Performances of surface emg and ultrasound signals in recognizing finger motion," in Human System Interactions (HSI), 2016 9th International Conference on. IEEE, 2016, pp. 117122.

[7] J. Rafiee, M. A. Rafiee, F. Yavari, and M. P. Schoen, "Feature extraction of forearm emg signals for prosthetics," Expert. Syst. Appl., vol. 38, no. 4, pp. 4058-4067, 2011.

[8] S. Shin, R. Langari, and R. Tafreshi, "A Performance Comparison of EMG Classification Methods for Hand and Finger Motion," in ASME 2014 Dynamic Systems and Control Conference. American Society of Mechanical Engineers, 2014, pp. V002T16A008-V002T16A008.
[9] M. Atzori, A. Gijsberts, I. Kuzborskij, S. Elsig, A.-G. Mittaz Hager, O. Deriaz, C. Castellini, H. Muller, and B. Caputo, "Characterization of a Benchmark Database for Myoelectric Movement Classification," IEEE Transactions on Neural Systems and Rehabilitation Engineering, vol. 23, no. 1, pp. 73-83, Jan. 2015.

[10] A. Shafti, R. R. Manero, A. Borg, K. Althoefer, and M. J. Howard, "Designing embroidered electrodes for wearable surface electromyography," in Robotics and Automation (ICRA), 2016 IEEE International Conference on. IEEE, 2016, pp. 172-177.

[11] Z. Ju, H. Liu, X. Zhu, and Y. Xiong, "Dynamic Grasp Recognition Using Time Clustering, Gaussian Mixture Models and Hidden Markov Models," Advanced Robotics, vol. 23, no. 10, pp. 1359-1371, Jan. 2009.

[12] Z. Ju, G. Ouyang, M. Wilamowska-Korsak, and H. Liu, "Surface EMG Based Hand Manipulation Identification Via Nonlinear Feature Extraction and Classification," IEEE Sensors Journal, vol. 13, no. 9, pp. 3302-3311, Sep. 2013.

[13] N. Jiang, K. B. Englehart, P. Parker et al., "Extracting simultaneous and proportional neural control information for multiple-dof prostheses from the surface electromyographic signal," Biomedical Engineering, IEEE Transactions on, vol. 56, no. 4, pp. 1070-1080, 2009.

[14] L. Pan, D. Zhang, J. Liu, X. Sheng, and X. Zhu, "Continuous estimation of finger joint angles under different static wrist motions from surface EMG signals," Biomedical Signal Processing and Control, vol. 14, pp. 265-271, Nov. 2014.

[15] M. Hioki and H. Kawasaki, "Estimation of finger joint angles from semg using a recurrent neural network with time-delayed input vectors," in Rehabilitation Robotics, 2009. ICORR 2009. IEEE International Conference on. IEEE, 2009, pp. 289-294.

[16] J. Ngeo, T. Tamei, and T. Shibata, "Continuous estimation of finger joint angles using muscle activation inputs from surface emg signals," in Engineering in Medicine and Biology Society (EMBC), 2012 Annual International Conference of the IEEE. IEEE, 2012, pp. 2756-2759.

[17] R. J. Smith, F. Tenore, D. Huberdeau, R. E. Cummings, and N. V. Thakor, "Continuous decoding of finger position from surface emg signals for the control of powered prostheses," in Engineering in Medicine and Biology Society, 2008. EMBS 2008. 30th Annual International Conference of the IEEE. IEEE, 2008, pp. 197-200.

[18] K. B. Kiesel, T. L. Uhl, F. B. Underwood, D. W. Rodd, and A. J. Nitz, "Measurement of lumbar multifidus muscle contraction with rehabilitative ultrasound imaging," Manual therapy, vol. 12, no. 2, pp. 161-166, 2007.

[19] Y. Zheng, M. Chan, J. Shi, X. Chen, and Q. Huang, "Sonomyography: Monitoring morphological changes of forearm muscles in actions with the feasibility for the control of powered prosthesis," Medical Engineering \& Physics, vol. 28, no. 5, pp. 405-415, Jun. 2006.

[20] J. Shi, Y. Zheng, X. Chen, and Q. Huang, "Assessment of muscle fatigue using sonomyography: Muscle thickness change detected from ultrasound images," Medical Engineering \& Physics, vol. 29, no. 4, pp. 472-479, May 2007.

[21] J. Shi, J.-Y. Guo, S.-X. Hu, and Y.-P. Zheng, "Recognition of Finger Flexion Motion from Ultrasound Image: A Feasibility Study," Ultrasound in Medicine \& Biology, vol. 38, no. 10, pp. 1695-1704, Oct. 2012.

[22] S. Sikdar, H. Rangwala, E. B. Eastlake, I. A. Hunt, A. J. Nelson, J. Devanathan, A. Shin, and J. J. Pancrazio, "Novel Method for Predicting Dexterous Individual Finger Movements by Imaging Muscle Activity Using a Wearable Ultrasonic System," IEEE Transactions on Neural Systems and Rehabilitation Engineering, vol. 22, no. 1, pp. 69-76, Jan. 2014.

[23] Y. Li, K. He, X. Sun, and H. Liu, "Human-machine interface based on multi-channel single-element ultrasound transducers: A preliminary study," in e-Health Networking, Applications and Services (Healthcom), 2016 IEEE 18th International Conference on. IEEE, 2016, pp. 1-6.

[24] N. Akhlaghi, C. Baker, M. Lahlou, H. Zafar, K. Murthy, H. Rangwala, J. Kosecka, W. Joiner, J. Pancrazio, and S. Sikdar, "Real-time Classification of Hand Motions using Ultrasound Imaging of Forearm Muscles," IEEE Transactions on Biomedical Engineering, pp. 1-1, 2015.

[25] C. Castellini and D. S. González, "Ultrasound imaging as a humanmachine interface in a realistic scenario," in Intelligent Robots and Systems (IROS), 2013 IEEE/RSJ International Conference on. IEEE, 2013, pp. 1486-1492.

[26] J.-Y. Guo, Y.-P. Zheng, L. P. Kenney, A. Bowen, D. Howard, and J. J. Canderle, "A comparative evaluation of sonomyography, electromyography, force, and wrist angle in a discrete tracking task," Ultrasound in medicine \& biology, vol. 37, no. 6, pp. 884-891, 2011.

[27] J. Shi, Y.-P. Zheng, Q.-H. Huang, and X. Chen, "Continuous monitoring of sonomyography, electromyography and torque generated by normal 
upper arm muscles during isometric contraction: sonomyography assessment for arm muscles," Biomedical Engineering, IEEE Transactions on, vol. 55, no. 3, pp. 1191-1198, 2008.

[28] V. Ravindra and C. Castellini, "A comparative analysis of three noninvasive human-machine interfaces for the disabled," Frontiers in neurorobotics, vol. 8, 2014.

[29] P. Stoica and R. L. Moses, Spectral analysis of signals. Pearson/Prentice Hall Upper Saddle River, NJ, 2005.

[30] B. Hudgins, P. Parker, and R. N. Scott, "A new strategy for multifunction myoelectric control," Biomedical Engineering, IEEE Transactions on, vol. 40, no. 1, pp. 82-94, 1993.

[31] K. Englehart, B. Hudgins, P. A. Parker, and M. Stevenson, "Classification of the myoelectric signal using time-frequency based representations," Medical engineering \& physics, vol. 21, no. 6, pp. 431-438, 1999.

[32] M. A. Oskoei and H. Hu, "Support vector machine-based classification scheme for myoelectric control applied to upper limb," Biomedical Engineering, IEEE Transactions on, vol. 55, no. 8, pp. 1956-1965, 2008.

[33] T. R. Farrell and R. F. Weir, "The optimal controller delay for myoelectric prostheses," Neural Systems and Rehabilitation Engineering, IEEE Transactions on, vol. 15, no. 1, pp. 111-118, 2007.

[34] A. Stango, F. Negro, and D. Farina, "Spatial correlation of high density emg signals provides features robust to electrode number and shift in pattern recognition for myocontrol," Neural Systems and Rehabilitation Engineering, IEEE Transactions on, vol. 23, no. 2, pp. 189-198, 2015.

[35] J. M. Hahne, B. Graimann, and K.-R. Müller, "Spatial filtering for robust myoelectric control," Biomedical Engineering, IEEE Transactions on, vol. 59, no. 5, pp. 1436-1443, 2012.

[36] J. Shi, S. Zhou, X. Liu, Q. Zhang, M. Lu, and T. Wang, "Stacked deep polynomial network based representation learning for tumor classification with small ultrasound image dataset," Neurocomputing, vol. 194, pp. 87-94, 2016.

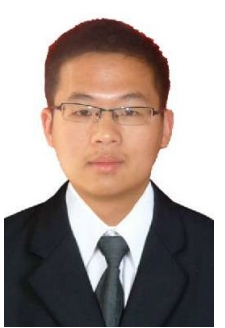

Youjia Huang Youjia Huang received the Master's degree from the School of Mechanical Engineering, Shanghai Jiao Tong University, Shanghai, China, in 2017 and the Bachelor's degree from the School of Mechanical Engineering, Jilin University, Changchun, China in 2014

His research interest lies in machine learning and biological signal processing on hand gesture classification.

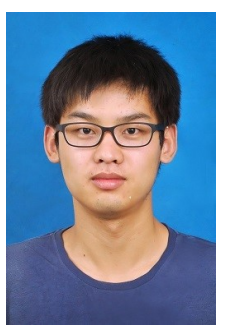

Xingchen Yang received the Bachelor's degree in mechanical engineering from Tianjin University, Tianjin, Shanghai, China, in 2015. He is currently pursuing the Ph.D. degree in the School of Mechanical Engineering, Shanghai Jiao Tong University, Shanghai, China.

His research interests include biological signal processing and machine learning.

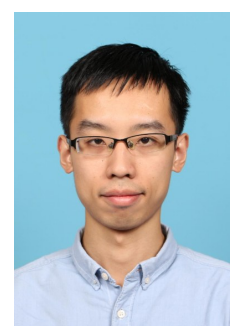

Yuefeng $\mathbf{L i}$ received the Master's degree from the School of Mechanical Engineering, Shanghai Jiao Tong University, Shanghai, China, in 2017.

His research interest lies in ultrasound signal processing on hand gesture and prediction and the development of the ultrasound transducers.

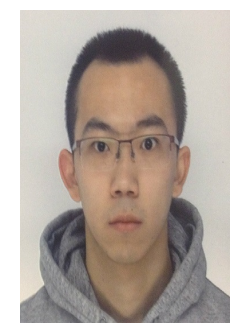

Dalin Zhou received the B.S. degree in automation from the University of Science and Technology of China, in 2012. He is currently pursuing the Ph.D. degree with the Intelligent System and Biomedical Robotics Group, University of Portsmouth, Portsmouth, U.K.

His research interests are signal processing and machine learning.



Keshi He received the Ph.D. degree from the School of Electronic Information and Electrical Engineering, Shanghai Jiao Tong University, Shanghai, China, 2015. He is currently working as a postdoc at the Robotic Institute, Shanghai Jiao Tong University, Shanghai, China.

His research interests include machine learning, image processing, and biological signal processing with application to upper limb prosthesis control.

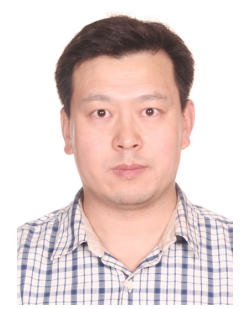

Honghai Liu received the Ph.D. degree in robotics from King's College London, London, U.K., in 2003.

$\mathrm{He}$ is the Chair Professor of Intelligent Systems and Robotics, University of Portsmouth, Portsmouth, U.K. His research interests include biomechatronics, pattern recognition, intelligent video analytics, intelligent robotics, and their practical applications with an emphasis on approaches that could make contribution to the intelligent connection of perception to action using contextual information.

Prof. Liu is a fellow of the Institution of Engineering and Technology. He is an Associate Editor of the IEEE TRANSACTIONS ON HUMAN MACHINE SYSTEMS, IEEE TRANSACTIONS ON INDUSTRIAL ELECTRONICS, and IEEE TRANSACTIONS ON INDUSTRIAL INFORMATICS. 